\title{
Hairy Black Holes in Massive Gravity: Thermodynamics and Phase Structure
}

\author{
Fabio Capela \\ Service de Physique Théorique, Université Libre de Bruxelles (ULB), \\ CP225 Boulevard du Triomphe, B-1050 Bruxelles, Belgium \\ and Germano Nardini \\ Fakultät für Physik, Universität Bielefeld, \\ Universitätsstraße 25, D-33615 Bielefeld, Germany
}

\begin{abstract}
The thermodynamic properties of a static and spherically symmetric hairy black hole solution arising in massive gravity with spontaneous Lorentz breaking are investigated. The analysis is carried out by enclosing the black hole in a spherical cavity whose surface is maintained at a fixed temperature $T$. It turns out that the ensemble is well-defined only if the "hair" parameter $Q$ characterizing the solution is conserved. Under this condition we compute some relevant thermodynamic quantities, such as the thermal energy and entropy, and we study the stability and phase structure of the ensemble. In particular, for negative values of the hair parameter, the phase structure is isomorphic to the one of Reissner-Nordstrom black holes in the canonical ensemble. Moreover, the phase-diagram in the plan $(Q, T)$ has a line of first-order phase transition that at a critical value of $Q$ terminates in a second-order phase transition. Below this line the dominant phase consists of small, cold black holes that are long-lived and may thus contribute much more to the energy density of the Universe than what is observationally allowed for radiating black holes.
\end{abstract}

\section{INTRODUCTION}

The discovery of the accelerated expansion of the Universe [1] has revived interest in the theories of gravity that can explain such observation without invoking dark energy. One class of these models is called "massive gravity". Models of massive gravity fulfilling Lorentz invariance have been recently constructed [2] and proved to be free from ghosts and instabilities at full non-perturbative level [3, 4. However, there are very few known solutions for such models. Instead, models of massive gravity with Lorentz violation are better understood at present. In these scenarios the spontaneous breaking of Lorentz symmetry is achieved by scalar fields that are coupled to gravity in a covariant way through derivative couplings 5 - 7 . As a consequence of this interaction, Lorentz violation is transmitted to the gravitational sector and the graviton becomes massive [6 8 . In a wide region of the parameter space these Lorentz breaking models are, at perturbative level, free from ghosts and tachyonic instabilities around Minkowski 8 and curved backgrounds 9]. They can also exhibit infrared modifications of the gravitational behavior 9]. Moreover, they are expected to reach the strong coupling regime at energies that are much higher than in Lorentz-invariant models of massive gravity [6] 9], even though it has not yet been rigorously proved. Interestingly, these Lorentz breaking theories are formulated in a non-perturbative way, making the study of black holes possible [10 13]. On top of that, black hole solutions are far richer than in General Relativity (GR) due to the presence of "hair" parameters [11, 12].

In GR the existence of black hole solutions raises questions of consistency with the general laws of thermodynamics. For instance, it has been argued 14 that black holes have temperature and entropy. The same conclu- sion was apparently reached by means of path integral methods [15]. However, the proof turned out not to be fully consistent because of a technical subtlety: the path integral approach requires black holes in thermal equilibrium with its surroundings and this situation is never fulfilled by Schwarzschild solutions. Instead, antide-Sitter (AdS) black holes achieve thermal equilibrium and the path integral procedure proposed in Ref. [15] can be consistently applied 16. Moreover, it turns out that black holes are just one of the phases permitted in AdS space and first order transitions between a black hole and globally-AdS spacetime may occur ${ }^{1}$. On the other hand, it might be expected that a similar result also arises for Schwarzschild black holes if one mimics the AdS cosmological constant by an infrared cutoff. In fact, as it was proven by York [19], after having enclosed the Schwarzschild black hole inside a spatial spherical surface at a fixed temperature, the configuration reaches thermal equilibrium and its phase structure turns similar to the AdS one.

It is interesting to understand whether one can apply the York's approach to investigate thermodynamics of hairy black hole solutions in Lorentz breaking massive gravity. This issue is the subject of the present paper and, as we will see, the conclusion depends on whether the black hole "hair" parameter $Q$ is conserved in the cavity. In particular, when $Q$ is assumed constant ${ }^{2}$, we obtain a rich thermodynamical phase structure that includes,

\footnotetext{
1 In the context of the AdS/CFT correspondence [17, this transition turns out to be dual to the confinement-deconfinement phase transition in large- $N$ gauge theories [18.

${ }^{2}$ In practice, we expect that the results obtained under this hypothesis can be extended to situations where $Q$ varies very slowly.
} 
for instance, first and second order phase transitions and phases consisting of stable and small, cold black holes (plausible dark matter candidates).

The paper is organized as follows. In Sect. II we briefly review the model of massive gravity and we sketch the eventual difficulties that may arise when applying the York's procedure. In Sect. III we review the static, spherically symmetric and asymptotically-flat black hole solution of massive gravity and we check whether it can be embedded in a spacetime that has a periodic Euclidean time coordinate and is bounded by a spatial sphere. This compatibility requirement is needed in order to apply the York's procedure, which we carry out in Sect. IV] assuming $Q$ to be conserved in the ensemble. In particular, we first regularize the black hole action by the "subtraction background" method [15], then we enclose the black hole solution in a sphere at a fixed temperature and, finally, we obtain the on-shell action of the ensemble. As we prove in Sect. V this procedure provides a well-defined ensemble that we use to investigate black hole thermodynamics. It turns out that, depending on the value of $Q$ inside the cavity, black holes can evolve in qualitatively different ways. In any case, their evolution always respects the Bekenstein-Hawking formula. In Sect. VI we relax the assumption concerning the conservation of $Q$ and we see that in such a case the York's procedure is inconsistent. Finally, Sect. VII is dedicated to summarize the main results of the paper and Appendix contains some analytic expressions valid for a specific choice of massive gravity parameters.

\section{MASSIVE GRAVITY AND THE SADDLE-POINT APPROXIMATION}

Massive gravity is conventionally described by the action $7+13$

$$
\begin{aligned}
I & =\int_{\mathcal{M}} d^{4} x \sqrt{g}\left[-\frac{1}{16 \pi} R+\Lambda^{4} \mathcal{F}\left(X, W^{i j}\right)\right] \\
& -\int_{\partial \mathcal{M}} d^{3} x \sqrt{\gamma} \frac{1}{8 \pi} K,
\end{aligned}
$$

with

$$
\begin{aligned}
X & =\Lambda^{-4} g^{\mu \nu} \partial_{\mu} \phi^{0} \partial_{\nu} \phi^{0}, \\
V^{i} & =\Lambda^{-4} \partial^{\mu} \phi^{i} \partial_{\mu} \phi^{0}, \\
W^{i j} & =\Lambda^{-4} \partial^{\mu} \phi^{i} \partial_{\mu} \phi^{j}-\frac{V^{i} V^{j}}{X},
\end{aligned}
$$

where Latin (Greek) indices run on space (spacetime) components. The first integral in Eq. (1) is evaluated on the manifold $\mathcal{M}$ with metric $g$ and contains two contributions: the usual Einstein-Hilbert term and a function $\mathcal{F}$ of four scalar fields $\phi^{\mu}$ that are minimally coupled to gravity by covariant derivatives. The second integral is instead the Gibbons-Hawking-York boundary term [15, 20, where $\gamma_{a b}$ is the metric induced on the boundary $\partial \mathcal{M}$ and $K$ is the trace of the extrinsic curvature $K_{i j}=\frac{1}{2} \gamma_{i}^{k} \nabla_{k} n_{j}$ of $\partial \mathcal{M}$ with unit normal $n^{i}$. Such a boundary term is required to have a well-defined variational principle in the presence of the border $\partial \mathcal{M}$.

The action (1) describes a low-energy effective theory valid below the ultraviolet cutoff $\Lambda$, which perturbative analyses estimate to be $\mathcal{O}\left(\sqrt{m M_{\mathrm{Pl}}}\right)$ [6], where $M_{\mathrm{Pl}}=$ $\sqrt{1 / 8 \pi}$ and $m$ are the Planck and the graviton masses, respectively. Its vacuum flat-spacetime solution has the form

$$
g_{\mu \nu}=\eta_{\mu \nu}, \quad \phi_{\text {flat }}^{0}=\Lambda^{2} t, \quad \phi_{\text {flat }}^{i}=\Lambda^{2} x^{i},
$$

which induces a spontaneous breaking of Lorentz symmetry. The background (2) preserves rotational symmetry when the function $\mathcal{F}$ is invariant under rotations in the internal space of the fields $\phi^{i}$. Moreover, the action (1) is invariant under the symmetry

$$
\phi^{i} \rightarrow \phi^{i}+\Theta^{i}\left(\phi^{0}\right)
$$

where $\Theta^{i}$ are arbitrary functions of $\phi^{0}$. This symmetry ensures that perturbations around the vacuum contain only two propagating degrees of freedom [7, corresponding to the two polarizations of a massive graviton.

To study the thermodynamics of this model, we use the Euclidean path integral

$$
\mathcal{Z}=\int \mathcal{D} g \mathcal{D} \phi \exp (-I[g, \phi])
$$

which is evaluated by integrating over all metrics and scalar fields satisfying particular boundary conditions. In the semi-classical limit $\mathcal{Z}$ is dominated by the stationary points of the action. This can be checked by expanding the path integral around a classical solution. Indeed, if the expansion provides a leading term that is finite, a linear term that vanishes on-shell, and a quadratic term that is positive definite, then the function $\mathcal{Z}$ can be expressed as

$$
\mathcal{Z} \approx \mathrm{e}^{-I\left[g_{\mathrm{cl}}, \phi_{\mathrm{cl}}\right]} \int \mathcal{D} \delta g \mathcal{D} \delta \phi \mathrm{e}^{-\delta^{2} I\left[g_{\mathrm{cl}}, \phi_{\mathrm{cl}} ; \delta g, \delta \phi\right]}
$$

and can be interpreted as the partition function of the model. However, such a derivation is not straightforward for the action (1) since the three properties listed above might not be fulfilled. In fact:

1. The on-shell leading term of the action diverges. This is a familiar problem in general relativity that is addressed using the "background subtraction" regularization technique [15. Following this subtraction scheme, we take the vacuum solution (2) as background $\left(g_{0}, \phi_{0}\right)$ and we consider $I_{E}$ as regularized action, defined as

$$
I_{E}(g, \phi) \equiv I(g, \phi)-I\left(g_{0}, \phi_{0}\right)
$$

In this way $I_{E}$ is finite for the class of fields $(g, \phi)$ that asymptotically approach the background $\left(g_{0}, \phi_{0}\right)$; 
2. The linear term may eventually not vanish for all perturbations around the classical solution. The non-vanishing behavior of such a term comes from the boundary contributions:

$$
\left.\delta I\right|_{\mathrm{cl}}=\int_{\partial \mathcal{M}} d^{3} x \sqrt{\gamma}\left[\pi^{a b} \delta \gamma_{a b}+\pi_{\mu}^{\phi} \delta \phi^{\mu}\right] .
$$

For the action (1) $\pi^{a b}$ and $\pi_{\mu}^{\phi}$ are given by

$$
\begin{aligned}
& \pi^{a b}=-\frac{1}{16 \pi}\left(K^{a b}-\gamma^{a b} K\right), \\
& \pi_{\mu}^{\phi}=2 \Lambda^{4} n_{\alpha}\left[\left(\frac{\partial \mathcal{F}}{\partial X}+\frac{\partial \mathcal{F}}{\partial W^{i j}} \frac{V^{i} V^{j}}{X^{2}}\right) \delta_{\mu}^{0} \partial^{\alpha} \phi^{0}\right. \\
& \left.+\frac{\partial \mathcal{F}}{\partial W^{i j}} \delta_{\mu}^{i} \partial^{\alpha} \phi^{j}-\frac{\partial \mathcal{F}}{\partial W^{i j}} \frac{V^{j}}{X} \partial^{\alpha}\left(\phi^{0} \delta_{\mu}^{i}+\phi^{i} \delta_{\mu}^{0}\right)\right]
\end{aligned}
$$

where $n_{\alpha}$ is the outward pointing unit normal to $\partial \mathcal{M}$. Therefore, some boundary conditions on the fields $g_{\mu \nu}$ and $\phi^{\mu}$ should be imposed in order to have a well-posed variational problem, i.e. $\left.\delta I\right|_{\mathrm{cl}}=0$;

3. The Gaussian integral in Eq. (5) corresponds to the one-loop contribution. Such contribution may diverge since the integration involves the determinant of second-order elliptic operators that cannot be regularized when negative eigenvalues are present. Indeed, if there exist negative eigenvalues, the density of states grows so rapidly that the ensemble turns out to be ill-defined and $\mathcal{Z}$ cannot be used to determine thermodynamical quantities. For this reason, in order to be able to study the thermodynamical properties of black holes, we need first to stabilize the ensemble. This can be performed as in Ref. [19]: we place the black hole inside a surface maintained at a fixed temperature.

In the next sections we explicitly show how to implement these three procedures for black hole solutions in massive gravity.

\section{THE BLACK HOLE SOLUTIONS}

By the coordinate transformations $r^{\prime} \rightarrow r=r\left(r^{\prime}\right)$ and $t^{\prime} \rightarrow t=t+\tau\left(r^{\prime}\right)$, the generic ansatz for the static spherically symmetric solution in Euclidean spacetime can be written as [11, 12 ]

$$
\begin{aligned}
d s^{2} & =\alpha(r) d t^{2}+\rho(r) d r^{2}+r^{2}\left(d \theta^{2}+\sin ^{2} \theta d \varphi^{2}\right) \\
\phi^{0} & =\Lambda^{2}[-i t+h(r)] \\
\phi^{i} & =\phi(r) \frac{\Lambda^{2} x^{i}}{r}
\end{aligned}
$$

For black hole solutions in massive gravity the explicit expression of this ansatz can be obtained by imposing Eqs. (10) to fulfill the equations of motions of the action (11). The black hole solution has then to be an extremum of the variation of the action with respect to the fields $g_{\mu \nu}$ and $\phi^{\mu}$ :

$$
\begin{aligned}
\delta I & =\int_{\mathcal{M}} d^{4} x \sqrt{g}\left[\mathbf{E}_{(1)}^{\mu \nu} \delta g_{\mu \nu}+\mathbf{E}_{\mu}^{(2)} \delta \phi^{\mu}\right] \\
& +\int_{\partial \mathcal{M}} d^{3} x \sqrt{\gamma}\left[\pi^{a b} \delta \gamma_{a b}+\pi_{\mu}^{\phi} \delta \phi^{\mu}\right]=0,
\end{aligned}
$$

where

$$
\begin{aligned}
& \mathbf{E}_{(1)}^{\mu \nu}=-\frac{1}{16 \pi}\left(R^{\mu \nu}-\frac{1}{2} R g^{\mu \nu}-8 \pi T_{\phi}^{\mu \nu}\right), \\
& T_{\phi}^{\mu \nu}=\frac{2 \Lambda^{4}}{\sqrt{g}} \frac{\delta\left(\sqrt{g} \mathcal{F}\left(X, W^{i j}\right)\right)}{\delta g_{\mu \nu}}, \\
& \mathbf{E}_{\mu}^{(2)}=-2 \Lambda^{4} \nabla_{\alpha}\left[\left(\frac{\partial \mathcal{F}}{\partial X}+\frac{\partial \mathcal{F}}{\partial W^{i j}} \frac{V^{i} V^{j}}{X^{2}}\right) \delta_{\mu}^{0} \partial^{\alpha} \phi^{0}\right. \\
& \left.+\frac{\partial \mathcal{F}}{\partial W^{i j}} \delta_{\mu}^{i} \partial^{\alpha} \phi^{j}-\frac{\partial \mathcal{F}}{\partial W^{i j}} \frac{V^{j}}{X} \partial^{\alpha}\left(\phi^{0} \delta_{\mu}^{i}+\phi^{i} \delta_{\mu}^{0}\right)\right] .
\end{aligned}
$$

For asymptotically-flat black holes the solution of Einstein and Goldstone's equations, respectively $\mathbf{E}_{(1)}^{\mu \nu}=0$ and $\mathbf{E}_{\mu}^{(2)}=0$, has a known analytical expression if the function $\mathcal{F}$ takes the form 12

$$
\mathcal{F}=\frac{12}{\lambda X}+6\left(\frac{2}{\lambda}+1\right) w_{1}-w_{1}^{3}+3 w_{1} w_{2}-2 w_{3}+12,
$$

where $\lambda$ is a positive constant and $w_{n}=\operatorname{Tr}\left(W^{n}\right)$. In such a case, for $\lambda \neq 1$, the ansatz (10) provides the black hole solution

$$
\begin{aligned}
& \alpha(r)=1-\frac{2 M}{r}-\frac{Q}{r^{\lambda}} \\
& \rho(r)=\frac{1}{\alpha(r)}, \\
& h(r)= \pm \int \frac{d r}{\alpha}\left[1-\alpha\left(\frac{Q}{12 m^{2}} \frac{\lambda(\lambda-1)}{r^{\lambda+2}}+1\right)^{-1}\right]^{1 / 2} \\
& \phi(r)=r
\end{aligned}
$$

which depends on the two arbitrary integration constants $M$ and $Q$. In the following we restrict our analysis to the class of solutions (14) with $\lambda>1$, so that the gravitational potential is asymptotically Newtonian and the parameter $M$ coincides with the Arnowitt-Deser-Misner (ADM) mass ${ }^{3}$. Moreover, we forbid naked singularities, i.e. $\alpha(r)$ must have real roots and the largest of them determines the radius of the event horizon. Depending on the signs and relative values of the parameter $M$ and $Q$, the following cases arise:

- $Q \geq 0$ and $M>0$ : The existence of the horizon is guaranteed: at all distances the gravitational potential is attractive and stronger than (or, for $Q=0$, equal to) the usual Schwarzschild black hole potential. Therefore $r_{+}$is never smaller than in the standard case.

3 These features have been discussed in Ref. 12] for $\lambda<1$. For the particular case $\lambda=1$, yielding a different solution from (14, see Appendix F of Ref. 11. Such a solution produces a divergent ADM mass . 
- $Q \geq 0$ and $M<0$ : The Newton's potential is repulsive at large distances and attractive near the horizon. This possibility is interesting because, even for $Q=0$, it does not have a corresponding case in $\mathrm{GR}^{4}$ Nonetheless, we do not analyze such negative mass configurations since they are incompatible with black hole thermodynamical laws [13]. As stated in Ref. 23, it seems likely that the cause of these incompatibilities is not Lorentz violation but the existence of negative energy states.

- $Q<0$ and $M>0$ : The horizon only exists when the condition

$$
2 M \geq \lambda|Q|^{1 / \lambda}\left(\frac{1}{\lambda-1}\right)^{\frac{\lambda-1}{\lambda}}
$$

is fulfilled. In this case the Newton's potential is always attractive until reaching the horizon but the attraction is weaker (which makes the event horizon radius $r_{+}$smaller) than in the Schwarzschild case.

\section{A. The boundary conditions}

As it has already been mentioned in Sect. II, the procedure to study equilibrium thermodynamics requires to enclose the asymptotically-flat black hole within a finite volume surface and then send the surface to infinity [19] (see Sect. IV for details). In the following, we consider a spherical cavity of radius $r_{\mathcal{B}}$ as the boundary and, to analyze the system at finite temperature, we impose periodicity on the Euclidean time. Due to the presence of this border, the black hole solution (14) must fulfill some boundary conditions in order to satisfy $\left.\delta I\right|_{\mathrm{cl}}=0$. The momentum $\pi_{0}^{\phi}$ conjugate to the scalar field $\phi^{0}$ on $\partial \mathcal{M}$ vanishes when evaluated at the solution (14). Then, there is no need to fix the scalar field $\phi^{0}$ at the boundary. Analogously, as a remnant of the internal spherical symmetry of the scalar fields $\phi^{i}$, i.e. $\left.\left(\phi^{i}\right)^{2}\right|_{\partial \mathcal{M}}=r_{\mathcal{B}}$, no boundary conditions emerge on the scalar sector. Instead, the momenta $\pi^{a b}$ conjugated to the induced metric $\gamma_{a b}$ are not null when evaluated at the background solution (14). For this reason we conclude that we only need to impose $\delta \gamma_{a b}=0$ to have vanishing boundary terms. Further conditions arise due to the compactification of

\footnotetext{
4 The choice $M<0$ is problematic in GR where: i) it leads to naked singularities; ii) it violates the null energy condition $T_{\mu \nu} k^{\mu} k^{\nu} \geq 0$ (being $k^{\mu}$ a future-pointing null vector field), which holds for the matter stress tensor 21] and implies the positivity of the ADM mass. Neither of the arguments exist in massive gravity: i) at short distance the repulsion changes to attraction, which creates the event horizon; ii) the stress-energy tensor of the scalar fields $T_{\mu \nu}^{\phi}$ does not satisfy the null energy condition, allowing for negative mass states to be constructed, e.g., as in the ghost condensate model [22].
}

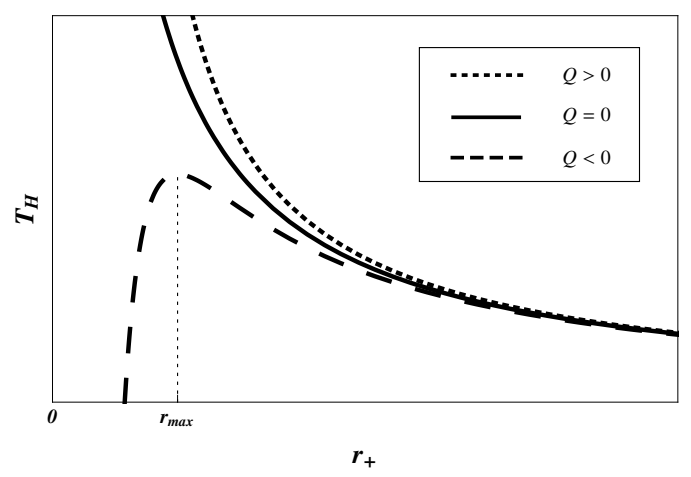

FIG. 1. The Hawking temperature $T_{H}$ as a function of the event horizon radius $r_{+}$for positive and negative scalar charge $Q$ and for the conventional Schwarzschild case $Q=0$. For $Q<0$ the Hawking temperature reaches a maximum at $r_{+}=$ $r_{\max } \equiv\left[\left(\lambda^{2}-1\right)|Q|\right]^{1 / \lambda}$.

the Euclidean time. Regularity of the metric at the horizon fixes the periodicity as $t \sim t+\beta_{H}$, where $\beta_{H}$ is related to the Hawking temperature $T_{H}$ by the equality

$$
T_{H}^{-1}=\beta_{H}=\left.\frac{4 \pi}{\partial_{r} \alpha}\right|_{r_{+}}=\frac{4 \pi r_{+}}{1+(\lambda-1) \frac{Q}{r_{+}^{\lambda}}} .
$$

Interestingly, the Hawking temperature behaves differently for positive and negative scalar charges (see Fig. 1). For $Q>0$ the temperature is larger than for $Q=0$ and decreases with the increasing of the event horizon radius $r_{+}$, as in the case of the conventional Schwarzschild black hole. For $Q<0$ the temperature is smaller than in the Schwarzschild case and its non-monotonic dependence on $r_{+}$reaches a maximum at $r_{+}=r_{\max } \equiv\left[\left(\lambda^{2}-1\right)|Q|\right]^{1 / \lambda}$. The periodicity of $t$ also imposes a periodicity on the scalar field $\phi^{0}(t, r) \sim \phi^{0}\left(t+\beta_{H}, r\right)$ which is linearly dependent on time. This does not provide any further constraint on the solution (14) since the periodicity of $\phi^{0}$ is allowed due to the presence of the global shift symmetry $\phi^{0} \rightarrow \phi^{0}+C$ in the action (1).

Notice that the Hawking temperature measured by an observer at the position $r$ is given by

$$
T(r)=\frac{\beta_{H}^{-1}}{\sqrt{\alpha(r)}}=\frac{1}{4 \pi r_{+}} \frac{\left(1+(\lambda-1) \frac{Q}{r_{+}^{\lambda}}\right)}{\sqrt{1-\frac{r_{+}}{r}+\frac{Q}{r_{+}^{\lambda-1} r}-\frac{Q}{r^{\lambda}}}} .
$$

In case of thermal equilibrium, setting the temperature measured at the surface $r=r_{\mathcal{B}}$ univocally determines the temperature of the configuration contained in the cavity. Once one has set $r_{\mathcal{B}}$ and $T\left(r_{\mathcal{B}}\right)$ the boundary $\partial \mathcal{M}$ is unambiguously fixed.

\section{THE ON-SHELL ACTION}

In this section we briefly review the background subtraction procedure needed to obtain a finite on-shell ac- 
tion. Subsequently, we compute the Euclidean action of the black hole solutions (14) in terms of the boundary conditions $\left(T\left(r_{\mathcal{B}}\right), r_{\mathcal{B}}\right)$ under the assumption that $Q$ is a conserved quantity in the cavity.

\section{A. The regularization procedure}

A priori, the Euclidean action (1) may present an integration problem at $r=r_{+}$and another at $r \rightarrow \infty$. The former is solved as usual by assigning the period $\beta_{H}$ to $t$, as in such a case the metric (14) extends smoothly onto the event horizon. The latter is instead more cumbersome. To regularize it [19, we perform the integration from $r=r_{+}$up to the infrared cutoff $r=r_{\mathcal{B}}$, we subtract off the action of the vacuum flat space (2) 5 , and finally we send $r_{\mathcal{B}}$ to infinity. In this way the Euclidean action is regularized and defined as in Eq. 66.

Notice that in a spherical cavity of finite volume the on-shell action (with the metric regularized at $r=r_{+}$) is always finite and, in addition, the thermodynamic stability is guaranteed [19]. Hence, as long $r_{\mathcal{B}}$ is finite, in principle there is no need to subtract any background to make the action finite. Instead, this procedure is necessary to have no divergent thermodynamical properties when the limit $r_{\mathcal{B}} \rightarrow \infty$ is taken. Then, in view of this limit, we apply the regularization procedure as a first step to analyze black hole thermodynamics.

\section{B. The regularized action for hairy black holes}

An apparent ambiguity may arise in the procedure to regularize the action (1): since the subtracted background (2) is regular everywhere, it does not require any specific periodicity of the time coordinate. However, black hole and background metrics have to match at the boundary surface $r=r_{\mathcal{B}}$. Thus, the time periodicity $\beta$ of the background has to be

$$
\beta=T\left(r_{\mathcal{B}}\right)^{-1},
$$

where $T\left(r_{\mathcal{B}}\right)^{-1}=\beta_{H} \sqrt{\alpha\left(r_{\mathcal{B}}\right)}$ as in Eq. (17).

Once the action (1) has been regularized, the evaluation of $I_{E}$ for the black hole solution (14) with radial integration $r_{+} \leq r \leq r_{\mathcal{B}}$ is straightforward. Indeed, as the metric (14) and $\mathcal{F}$ of Eq. (13) are stationary, the time integration in $I_{E}$ just gives rise to a multiplicative factor $\beta$. Moreover, the integration on the other variables can be performed in a closed manner. The regularized (quasi-local) action of the black hole then results to be

$$
I_{E}=\beta \mathcal{E}_{B Y}-\frac{1}{4} \mathcal{A}_{\mathrm{BH}}
$$

\footnotetext{
5 Of course, this subtracted background is not $\mathbb{R}^{4}$ but $\mathrm{S}^{1} \times \mathbb{R}^{3}$ since the periodicity of time is maintained.
}

with

$$
\begin{aligned}
\mathcal{A} & \equiv 4 \pi r_{+}^{2} \\
\mathcal{E}_{B Y} & =r_{\mathcal{B}}\left[1-\sqrt{\alpha\left(r_{\mathcal{B}}\right)}\right]=\frac{1}{8 \pi} \int_{\mathcal{B}_{\bar{t}}} d^{2} x \sqrt{\sigma}\left(k-k_{0}\right),
\end{aligned}
$$

where $k$ is the trace of the extrinsic curvature of the twoboundary $\left.\mathcal{B}_{\bar{t}} \equiv \partial \mathcal{M}\right|_{t=\bar{t}}, \sigma$ is the induced metric on $\mathcal{B}_{\bar{t}}$ and $k_{0}$ refers to the extrinsic curvature of $\mathcal{B}_{\bar{t}}$ embedded in the vacuum space (2).

The last equality in Eqs. 20 shows explicitly that $\mathcal{E}_{B Y}$ is the Brown-York quasi-local energy [24, corresponding to the Hamiltonian that generates the time translation at the two-boundary $\mathcal{B}_{\bar{t}}$. For this reason we can interpret $\mathcal{E}_{B Y}$ in Eq. (19) as the energy of the black hole ${ }^{6}$ On the other hand, plugging Eq. 114) in Eq. 20 reads

$$
M=\mathcal{E}_{B Y}-\frac{\mathcal{E}_{B Y}^{2}}{2 r_{\mathcal{B}}}-\frac{Q}{2 r_{\mathcal{B}}^{\lambda-1}},
$$

showing that the ADM mass $M$ is the total energy of the black hole in the limit $r_{\mathcal{B}} \rightarrow \infty$. For this reason, when we send the cavity surface to infinity, the regularized on-shell action is given by

$$
I_{E}=\beta_{H} M-\frac{1}{4} \mathcal{A}_{\mathrm{BH}}
$$

which still vanishes when the event horizon goes to zero as it reproduces the action of the subtracted vacuum spacetime 7 A detailed discussion about the finite energy of the black hole solutions (14) can be found in Ref. 25.

\section{THERMODYNAMICS AND PHASE TRANSITIONS}

Eq. 17) evaluated at $r=r_{\mathcal{B}}$ produces a function of the ADM mass $M$ in terms of the parameters of the ensemble $\beta, r_{\mathcal{B}}$ and $Q$. Depending on the particular values of these parameters, there can exist zero, one or multiple black hole solutions that are allowed inside the cavity. When several configurations are permitted, phase transitions may occur. In the present section we analyze this issue under the hypothesis that the scalar charge is conserved inside the cavity (for considerations without this assumption see Sect. VI).

\section{A. The phases}

In order to determine the number of black hole solutions allowed inside a cavity containing a given scalar

${ }^{6}$ Remind that by construction $\mathcal{E}_{B Y}=0$ for the vacuum spacetime (2).

7 Of course, this conclusion is correct just for $M>0$, as we are assuming. 

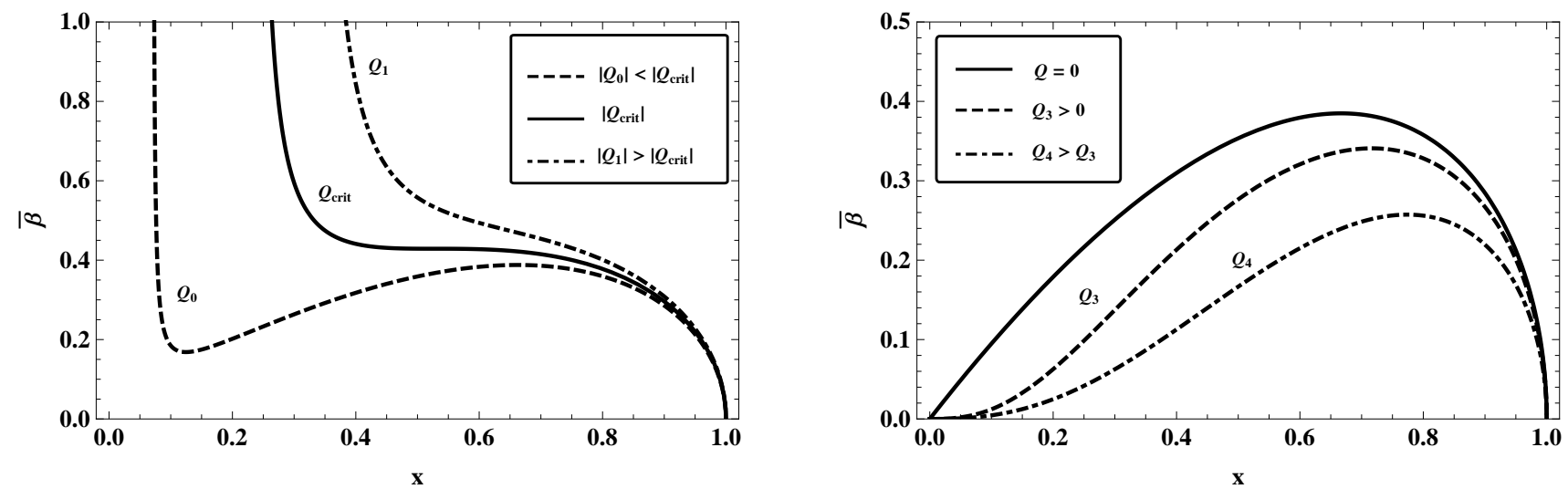

FIG. 2. Left panel: $\bar{\beta}$ as a function of the event horizon for negative scalar charges. Depending on the value of the scalar charge, the number of solutions is one or three. Right panel: $\bar{\beta}$ as a function of the event horizon for positive scalar charge. Depending on the value of $\bar{\beta}$, there exist two or no solutions. Negative slopes correspond to thermally stable states.

charge, we work out the temperature at the boundary, $\beta^{-1}$, as a function of $r_{+}$for fixed $Q$. This can be done by taking Eq. (17):

$$
\bar{\beta}(x, \mathcal{Q})=x \frac{\sqrt{1-x} \sqrt{1+\frac{\mathcal{Q}}{x^{\lambda}-1} \frac{1-x^{\lambda-1}}{1-x}}}{1+(\lambda-1) \frac{\mathcal{Q}}{x^{\lambda}}},
$$

where $x \equiv r_{+} / r_{\mathcal{B}}, \mathcal{Q} \equiv Q / r_{\mathcal{B}}^{\lambda}$ and $\bar{\beta} \equiv \beta / 4 \pi r_{\mathcal{B}}$. Observe that $\bar{\beta}$ positivity is guaranteed for $Q>0$ and is equivalent to require Eq. 15) for $Q<0$.

The plot of $\bar{\beta}(x, \mathcal{Q})$ is presented in Fig. 2 for specific values of $\mathcal{Q}$. The curves are obtained for a certain $\lambda$ but the general features of the solution are independent of this choice.

For the case $Q>0$ (right panel of Fig. 2 ) the function $\bar{\beta}(x, \mathcal{Q})$ exhibits a simple behavior: if $\bar{\beta}$ is below a certain value $\bar{\beta}_{\max }$, two black hole configurations with the same $Q$ are allowed; otherwise no black hole (with the assumed scalar charge) is permitted. Moreover, the value of $\bar{\beta}_{\text {max }}$ gets larger as $Q$ decreases and it is bounded by the relation $\bar{\beta}_{\max } \leq 2 / \sqrt{27}$ where the equality occurs for Schwarzschild black holes.

For the case $Q<0$ (left panel of Fig. 2) $\bar{\beta}$ exhibits three different qualitative behaviors. For $|\mathcal{Q}|>\left|\mathcal{Q}_{\text {crit }}\right|$ (with $\mathcal{Q}_{\text {crit }}<0$ quantified later) at each temperature there is one single solution. In particular, the event horizon of the black hole grows as the temperature and reaches the boundary only when $\bar{\beta}=0$. For $|\mathcal{Q}|<\left|\mathcal{Q}_{\text {crit }}\right|$ there is an interval of temperatures outside which $\bar{\beta}$ behaves as in the previous case, i.e. only large (nearly extremal) black holes exist at very high (very low) temperature. Instead, for $\bar{\beta}$ within this interval, three black hole configurations are allowed. These three solutions approach the same event horizon for $|\mathcal{Q}| \rightarrow\left|\mathcal{Q}_{\text {crit }}\right|^{-}$and for this reason $\left|\mathcal{Q}_{\text {crit }}\right|$ can be defined as the value of $\mathcal{Q}$ at which the extrema of $\bar{\beta}$, located at $\partial \bar{\beta} / \partial x=0$, coincide. For instance, when $\lambda=2$ it turns out to be (see Appendix for details)

$$
\left|\mathcal{Q}_{\text {crit }}\right|=\frac{1}{9+4 \sqrt{5}} .
$$

\section{B. The local stability}

The local stability in the saddle point approximation is related to the convergence of the integral in the on-shell partition function 26, as we briefly review now.

The on-shell partition function $\mathcal{Z}$ can be expressed as

$$
\mathcal{Z}=\int d E e^{-I(E)}=\int d E \nu(E) e^{-\beta E},
$$

where $\nu(E)$ is the density of classical states with energy $E$. Applying the saddle point approximation, which consists in expanding the action up to quadratic order around the stationary points $E_{\text {stat }}$, the integration in Eq. (25) can be evaluated and the result is finite only if $\left.\delta^{2} I\right|_{E_{\text {stat }}}>0$. On the other hand, in the saddle point approximation the energy and heat capacity can be approximated as $\langle E\rangle \equiv-\partial \ln \mathcal{Z} / \partial \beta \approx E_{\text {stat }}$ and $C \equiv \partial\langle E\rangle / \partial \beta^{-1} \approx \partial E_{\text {stat }} / \partial \beta^{-1}$ and, finally, it turns out to be

$$
C \approx \beta^{2}\left(\left.\delta^{2} I\right|_{E_{\text {stat }}}\right)^{-1} .
$$

Consequently, since a configuration of a thermodynamic system is locally stable when its heat capacity is positive, one concludes that the convergence of the integral in the partition function is equivalent to the thermal stability of the system 26 .

From this argument we can infer the stability property of the configurations depicted in Fig. 2, In fact, negative (positive) slopes of the isocharge curves $\bar{\beta}(x)$ are equivalent to positive (negative) heat capacity. Thus, for the 

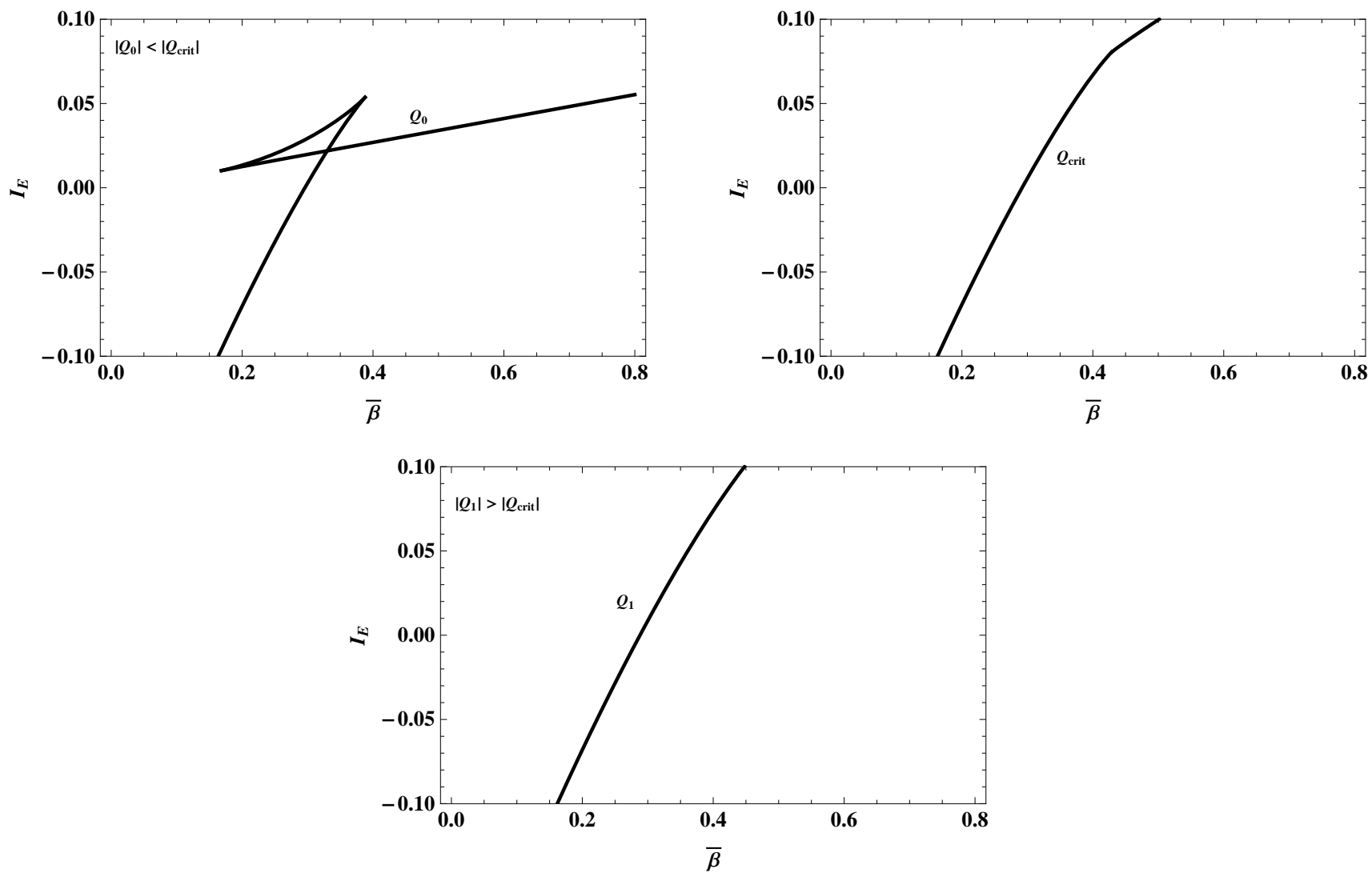

FIG. 3. The Euclidean action as a function of $\bar{\beta}$ for negative scalar charge in the three relevant cases. The values of $Q$ are the same as in Fig. 2

case $Q>0$ (right panel of Fig. 2), when two black hole solutions are allowed at a given temperature, the smallest is thermodynamically unstable and the largest is stable. Instead, for $Q<0$ (left panel of Fig. 2), when three black holes configurations with the same $\beta$ and $Q$ are possible, the smallest and largest are stable while the intermediate is unstable. This unstable solution thus corresponds to a maximum of the Euclidean action whose value is associated to the tunnelling rate between the two stable configurations [27].

Therefore, the result presented in Fig. 2 might have striking implications for cosmology. By taking $r_{+} \ll r_{\mathcal{B}}$ we expect to mimic the black hole conditions in the present Universe, since the Universe is much larger than any event horizon radius. In such a case Fig. 2 shows that black holes with $Q \geq 0$ have high temperature and tend to evaporate. Their relic abundance is then constrained by the usual astrophysical bounds valid for radiating black holes 28 . On the contrary, black holes with $Q<0$ are cold long-lived configurations, which in general are poorly constrained and may be plausible dark matter candidates 28]. Of course, the discovery of such stable objects would be an important finding in favor of massive gravity. Moreover, even though massive gravity can qualitatively explain either dark energy and dark matter by tuning the gravitational force at long and intermediate distances 29, the presence of black hole energy density might be a fundamental ingredient to fit the data. As a last remark, we highlight that for locally-stable black hole configurations, the action $I_{E}$ fulfills the three requirements of Sect. III. Thus, for such configurations $\mathcal{Z}$ can be interpreted as the partition function of the system. From Eq. 22) one can therefore compute thermodynamical quantities as, for instance, the entropy:

$$
S=\beta\left(\frac{\partial I_{E}}{\partial \beta}\right)-I_{E}=\frac{1}{4} \mathcal{A}_{\mathrm{BH}}
$$

which is the usual Bekenstein-Hawking formula [14 and agrees with the result found in Ref. [13, by means of Wald's formula [30. Hence, the Euclidean action reduces to the free energy in the semiclassical approximation, i.e. $I_{E}=\beta F$ with $F=M-T_{H} S$, so that the first law of black hole thermodynamics is recovered by requiring $F$ to have a minimum. Moreover, as demonstrated in Ref. 31] for static spherically symmetric gravitational systems, the thermal energy is identical to the quasilocal energy:

$$
\mathcal{E}_{B Y}=\langle E\rangle=\frac{\partial I_{E}}{\partial \beta}
$$



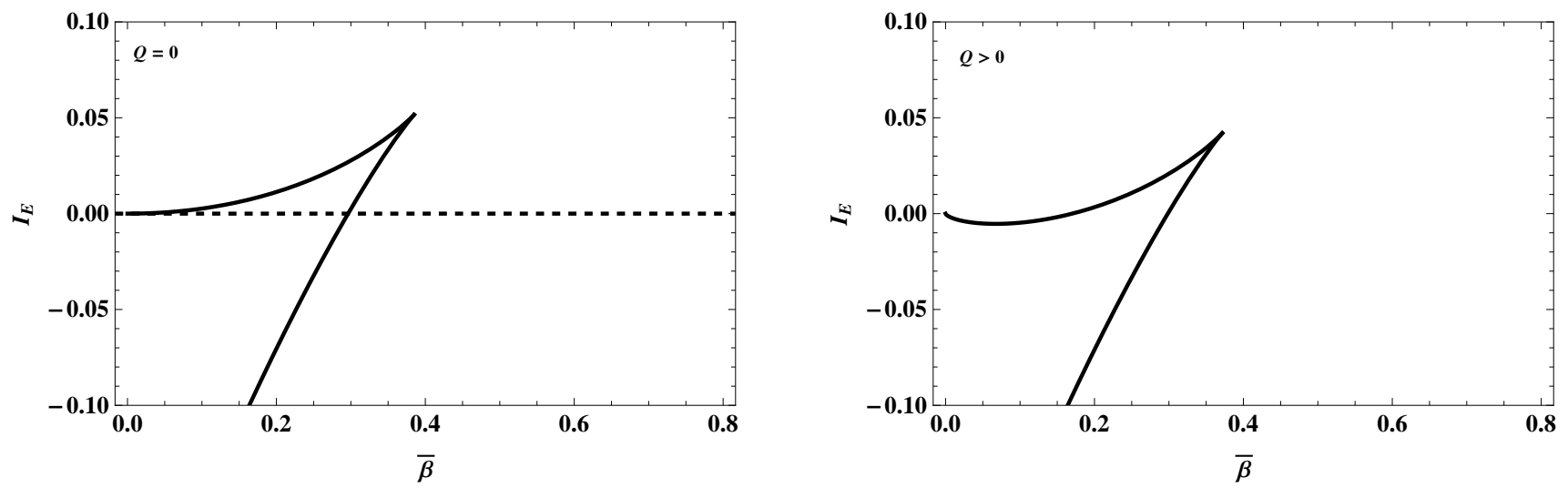

FIG. 4. The Euclidean action as a function of $\bar{\beta}$ in the case $Q=0$ (left panel) and $Q>0$ (right panel). The solid and dashed lines correspond to a black hole and vacuum phase, respectively.

\section{Global stability, phase structure and critical behavior}

In the previous section we have determined when the black hole solutions (14) are locally stable. In particular, we found that under certain conditions multiple locallystable solutions are allowed. However, for some fixed values of $Q$ and $\beta$, only one of these solutions can be globally stable (i.e. it corresponds to the global minimum of $I_{E}$ ) while the others have to be metastable (i.e. they are local but not global minima of $I_{E}$ ).

In order to make manifest the phase structure of the black hole solutions (14), we analyze $I_{E}$ as a function of temperature for a fixed $\mathcal{Q}$. The result is shown in Fig. 3 and 4 , respectively for negative and positive scalar charge. To read off the informations the figures contain, it can be useful to take into account also Fig. 2. When $Q$ is negative and $|\mathcal{Q}| \geq\left|\mathcal{Q}_{\text {crit }}\right|$ (top right and bottom panels of Fig. 3), there is only one solution independently of the specific temperature, as we already inferred from Fig. 2. Instead, when $Q$ is negative but $|\mathcal{Q}|<\left|\mathcal{Q}_{\text {crit }}\right|$ (top left panel of Fig. 3), the competition between the black hole phases is more curious. Starting at very low temperature and then heating up the system we see that at the beginning only one solution exists. This phase remains globally stable up to the critical temperature $\bar{\beta}_{c}^{-1}$ $\left(\bar{\beta}_{c} \simeq 0.33\right.$ in the example of the Fig. 2 and 3 above which it becomes metastable. Subsequently, a first order phase transition may occur and, interestingly, above a certain temperature $(\bar{\beta} \lesssim 0.17$ in the example) it has to. This property of the phase transition holds also starting at high temperature and then cooling down the system 8 Such a phase structure is isomorphic to the case of

\footnotetext{
8 This feature might have interesting applications, as for instance in Randall-Sundrum or QCD models, which typically suffer from a too long-lived metastable phase [32].
}

Reissner-Nordstrom black hole in AdS 33 and in a cavity 34 for the canonical ensemble (fixed electric charge). To test the similarity, we examine in detail the behavior of our case at $\mathcal{Q}=\mathcal{Q}_{\text {crit }}$ near the critical temperature $T_{c}=\bar{\beta}_{c}^{-1}$. The entropy and the heat capacity scale as (see Appendix for details)

$$
\begin{aligned}
S-S_{\mathrm{c}} & \propto\left(T-T_{\mathrm{c}}\right)^{1 / 3}, \\
C & \propto\left(T-T_{\mathrm{c}}\right)^{-2 / 3},
\end{aligned}
$$

implying that there is a second order phase transition at the critical temperature $T_{c}$. The value of the critical exponent of the heat capacity has thus the same value as for Reissner-Nordstrom black holes in AdS, dS and flat space in a cavity at fixed electric charge [33 35. Such a universal behavior is remarkable, knowing that the Euclidean action is different.

In some sense, the phase structure for $Q<0$ and $|\mathcal{Q}|<\left|\mathcal{Q}_{\text {crit }}\right|$ looks like the one for $Q=0$ (left panel of Fig. 4) apart from two main differences: i) at high temperature the allowed phase is not a black hole solution but the vacuum spacetime (2) (dashed line in the figure); ii) starting at low temperatures and then heating up the system, the first order phase transition (possible at $\bar{\beta} \lesssim 0.3$ in the figure) is not always guaranteed since the vacuum phase is allowed at high temperature 9 Instead, for $Q>0$ (right panel of Fig. 4) the phase structure is different: no phase is allowed at low temperature $\left(\bar{\beta}>\bar{\beta}_{\max } \simeq 0.38\right.$ in the example). A possible explanation of this result might be that at low temperature the existing phase is a state that has scalar charge and no event horizon. This could perfectly be a star with low free energy [11, 12, which could not emerge from

9 Although the presence of the scalar fields, such a phase structure is very similar to the one arising in GR for Schwarzschild black holes enclosed in a cavity [19] and Schwarzschild-AdS black holes [16]. 

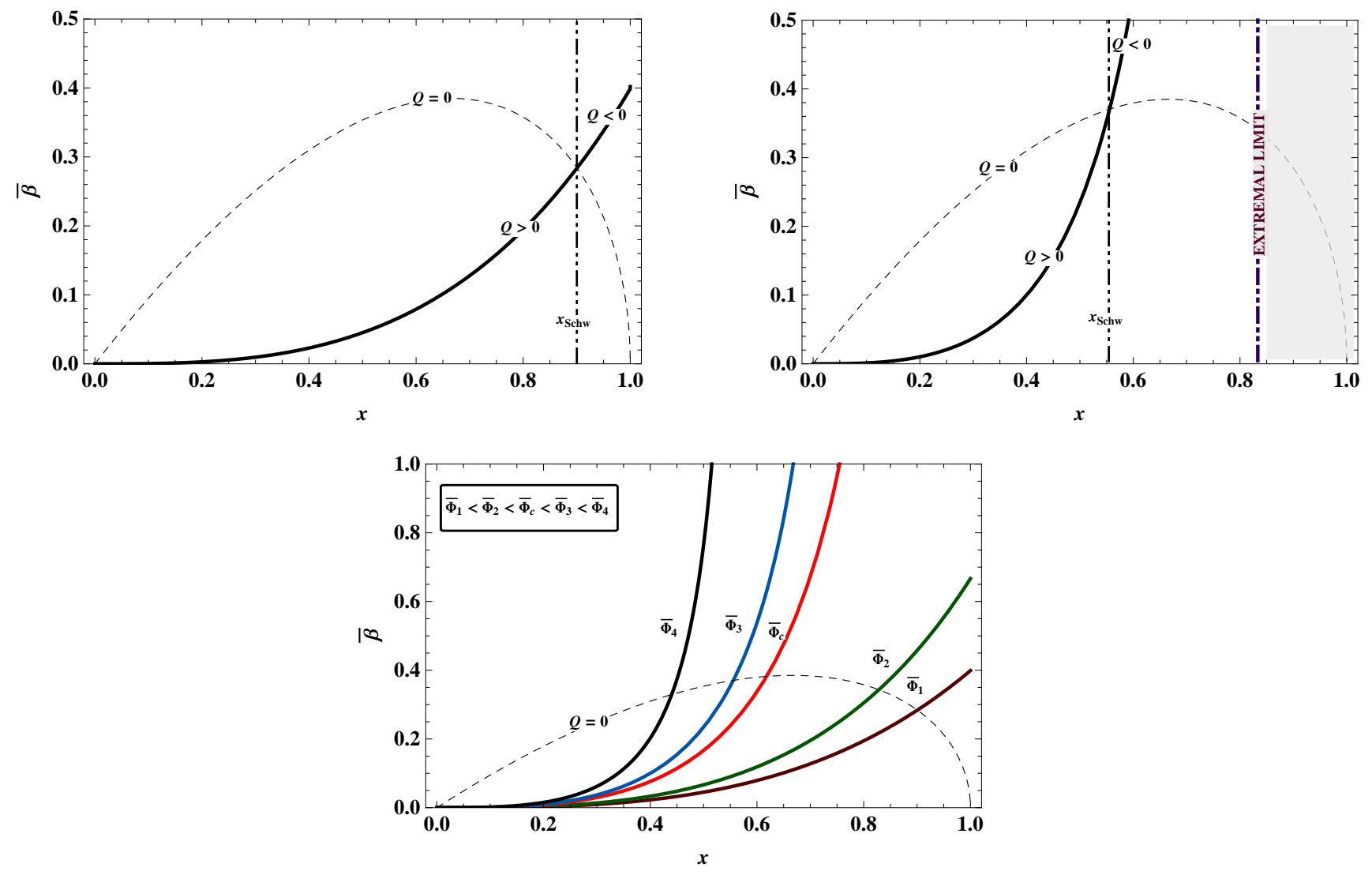

FIG. 5. Top left panel: $\bar{\beta}$ as a function of the event horizon for a particular value of $\bar{\Phi}<\bar{\Phi}_{\mathrm{c}}$. At $x=x_{\text {Schw }}$ the scalar charge of the solution changes of sign. Top right panel: $\bar{\beta}$ as a function of the event horizon for a value of $\bar{\Phi}>\bar{\Phi}_{\mathrm{c}}$. The black hole never engulfs the cavity and tends to the extremal limit at low temperatures. For $x$ higher than the extremal limit, the configuration has no event horizon. Bottom panel: $\bar{\beta}$ as a function of the event horizon for different values of $\bar{\Phi}$.

our analysis. On the other hand, one might guess that this peculiarity arises because we are assuming that $Q$ is conserved. In the next section, we assume other boundary conditions where the scalar charge is allowed to vary inside the cavity.

\section{OTHER BOUNDARY CONDITIONS}

In principle, one can assume the scalar charge not to be conserved inside the cavity. In such a case the phase evolution is no longer constrained by the initial value of $Q$ but by the value of $\Phi$, the scalar-charge "potential" at the surface of the cavity. In this ensemble it is straight- forward to see that the Euclidean action compatible with the regularity condition (17) is given by

$$
I_{E}=\beta\left(\mathcal{E}_{B Y}+\Phi Q\right)-\frac{1}{4} \mathcal{A}_{\mathrm{BH}}
$$

where

$$
\Phi=\frac{1}{2 \sqrt{\alpha\left(r_{\mathcal{B}}\right)}}\left[r_{+}^{1-\lambda}-r_{\mathcal{B}}^{1-\lambda}\right] .
$$

One may prove that the present ensemble is always dominated by a configuration with a negative heat capacity and is therefore ill-defined, as we shortly see now.

By combining Eqs. (18) and (32), $\beta$ can be expressed as function of $r_{+}$and $\Phi$. This relation can be more conveniently rewritten as

$$
\bar{\beta}=\frac{x^{2+\lambda}\left(1-x^{\lambda-1}\right)^{2} \bar{\Phi}}{x^{2}(-1+\lambda)-2 x^{1+\lambda}(-1+\lambda)-x^{3 \lambda} \bar{\Phi}^{2}+x^{2 \lambda}\left(-1+\lambda+\bar{\Phi}^{2}+(-1+x) \lambda \bar{\Phi}^{2}\right)},
$$


for several values of $\Phi$. The scalar charge changes along the curve and it is positive (negative) when the solution is below (above) the dashed line. The radius at which this cross occurs is marked as $x_{\text {schw }}$ in Fig. 5 (top panels, the left plot being for $\bar{\Phi}<\bar{\Phi}_{c}$ and the right one for $\bar{\Phi}>\bar{\Phi}_{c}$ ). Furthermore, when $\bar{\Phi}>\bar{\Phi}_{\mathrm{c}}$, the black hole event horizon radius is bounded from above by the extremal case limit and can never reach the spherical cavity (see Appendix for some analytic results).

The behavior of $\bar{\beta}$ described above seems problematic since the positiveness of the heat capacity depends on the sign of the slope of $\bar{\beta}$. For a positive slope, as in the case here, the heat capacity is negative. As we have discussed in Sect. $\mathrm{VB}$ this necessarily implies a divergent integral for the partition function, i.e. the partition function in the saddle point approximation is not well-defined and produces an imaginary result. This may be interpreted as the effect of an unidentified metastable phase. However, the present framework does not offer an unambiguous description and such interpretation should be taken with precaution.

\section{CONCLUSIONS}

Lorentz breaking massive gravity is an interesting theory that may explain the recent acceleration of the Universe without invoking dark energy. It also provides peculiar black hole solutions due to the presence of hair parameters that modifies the standard gravitational potential. In this theory the analog of the Schwarzschild black hole - the asymptotically flat spherically symmetric solution - depends on two parameters: the mass and the "scalar charge" (characterizing the hair strength). In this paper we have analyzed equilibrium states and phase structures of such a solution enclosed in a spherical surface kept at a fixed temperature.

We have proven that when the scalar charge inside the cavity is not conserved, the ensemble is ill-defined. On the contrary, when the scalar charge is held fixed, the saddle point approximation can be applied to obtain the partition function $\mathcal{Z}=e^{-I_{E}}$, where $I_{E}$ is the regularized on-shell Euclidean action. With that formalism we were able to study the black hole thermodynamics and phase structure. In particular, the black hole entropy follows the Bekenstein-Hawking formula.

Depending on the value of the scalar charge $Q$ contained in the cavity, the phase structure presents completely different behaviors that can be summarized as follows (being $Q_{\text {crit }}$ a critical value of the scalar charge):

- $Q>0$ : Above a certain temperature there exist two black hole solutions with different event horizon radii. The smaller black hole is unstable while the larger is globally stable and describes the phase present in the cavity. Below that temperature we do not find any solution with event horizon, probably because the analysis is not suitable for this phase.
- $Q=0$ : Below a certain temperature no black hole solution exists and the phase consists of the usual globally-flat background of massive gravity. Above that temperature the previous phase competes with a new phase consisting of a large black hole.

- $Q_{\text {crit }}<Q<0$ : There is an interval of temperatures where there are three black holes solutions with different sizes of event horizon. The smallest and the largest are locally stable and therefore correspond to local minima of the free energy, but the intermediate is unstable as it corresponds to a maximum of the free energy. Hence, a tunnelling between the two stable phases may occur and this first order phase transition is forced to happen when the temperature crosses the whole interval.

- $Q=Q_{\text {crit }}$ : Only one black hole solution is allowed at each temperature. At a certain temperature a second-order phase transition happens and at this moment the critical exponent of the heat capacity is $-2 / 3$.

- $Q<Q_{\text {crit }}$ : There is a single (both globally and locally) stable black hole at every temperature.

Further investigations on the cases with negative $Q$ are worthwhile. Indeed, their isomorphism to ReisserNordstrom black holes in AdS, dS and flat space in the canonical ensemble 33 35 is peculiar, knowing that their Euclidean actions are different. Furthermore, at low temperature the globally stable configurations are small cold black holes. These objects overcome most of the astrophysical constraints [28 and, in principle, might be the dark matter candidates of massive gravity. Dedicated dark matter analyses would be needed to check this possibility but, as a first step, one should understand whether the black hole scalar charge is actually conserved (or at least varies very slow) in nature. In order to address this question one should probably comprehend the origin of the modified character of black hole horizons [10, 36, which is hard to understand without a known UV completion of the theory. However, with the use of the AdS/CFT correspondence one may attempt to study such problems [37. In that context, the study of the phase structure may play an important role as a valuable test of the AdS/CFT correspondence. Since the phase structure emerging for AdS boundary conditions appears as well as for asymptotically-de Sitter black holes and asymptotically-flat black holes in cavities [35], one should expect the CFT side to have a rich variety of phases (such as deconfinement/confinement) dual to what has been studied in this paper. Moreover, Lorentz symmetry violation should arise in the CFT side 38. Trying to understand regimes in which Lorentz violation is sizeable will hopefully allow us to devise the right experimental tests to decide whether massive gravity is or is not realized in nature. 


\section{ACKNOWLEDGMENTS}

We are grateful to P. Tinyakov for stimulating discussions and useful advices. The work of F.C. is supported in part by the IISN, Belgian Science Policy (under contract IAP V/27) and by the "Action de Recherche Concertés" (ARC), project "Beyond Einstein: fundamental aspects of gravitational interactions" .

\section{Appendix A: Some analytic results for $\lambda=2$}

In this appendix, we consider the case $\lambda=2$. This particular choice allows us to determine the main feature of the general case by some analytic calculations. In Appendix A 1 we focus on the ensemble with fixed scalar charge $Q=Q_{\text {crit }}$ and in Appendix A 2 we determine the behavior of $\bar{\beta}$ for the ensemble with fixed scalar charge potential.

\section{Case with fixed scalar charge $Q$}

For negative values of the scalar charge the function $\bar{\beta}$ has two extrema (see left panel of Fig. 22): one maximum and one minimum. The location of such extrema is obtained by solving the equation

$$
5 \mathcal{Q}^{2}+(2-2 \mathcal{Q}-3 x) x^{3}-6 \mathcal{Q} x(-1+\mathcal{Q}+x)=0 \text {. }
$$

The critical scalar charge is obtained when both extrema are degenerate, i.e. when the discriminant of the polynomial equation A1 is vanishing. By performing the explicit computation, the discriminant of the equation (A1) takes the form:

$$
\triangle=-\xi_{0} \mathcal{Q}^{4} \sum_{n=3}^{9} \xi_{n} \mathcal{Q}^{n}
$$

where $\xi_{i}$ are real and positive constants. Therefore, several values for $\mathcal{Q}$ are allowed for a vanishing discriminant A2. However, only one value of $\mathcal{Q}$ corresponds to a positive event horizon radius:

$$
\mathcal{Q}_{\text {crit }}=\frac{1}{-9-4 \sqrt{5}} .
$$

By replacing the critical value of the scalar charge in Eq. A1, we obtain the critical value of the event horizon radius:

$$
x_{\text {crit }}=5-2 \sqrt{5} .
$$

By substituting both $x_{\text {crit }}$ and $\mathcal{Q}_{\text {crit }}$ in $\bar{\beta}$, we have the critical inverse temperature

$$
\bar{\beta}_{\text {crit }}=\frac{5}{2} \sqrt{85-38 \sqrt{5}} .
$$

We note that such critical values are similar to the Reissner-Nordstrom case 35 . At $\mathcal{Q}=\mathcal{Q}_{\text {crit }}$, we can expand $\bar{\beta}$ around the critical point $x_{\text {crit }}$ :

$$
\bar{\beta}-\bar{\beta}_{\text {crit }}=\left.\frac{1}{3 !} \frac{\partial^{3} \beta}{\partial x^{3}}\right|_{x=x_{\text {crit }}}\left(x-x_{\text {crit }}\right)^{3}+\ldots .
$$

The second derivative of $\bar{\beta}$ vanishes, when evaluated at the critical point. From here, one can very easily obtain the behavior of the entropy and heat capacity near the critical temperature:

$$
\begin{aligned}
S-S_{\text {crit }} & \propto\left(T-T_{\text {crit }}\right)^{1 / 3}, \\
C & \propto\left(T-T_{\text {crit }}\right)^{-2 / 3} .
\end{aligned}
$$

As we have already mentioned in Sect. VC, the critical exponent for the heat capacity has the same value $-2 / 3$ as for Reissner-Nordstrom black holes in AdS, dS and flat space in a cavity at fixed electric charge [33 35]. This implies that at the critical value of the scalar charge, we have a second order phase transition. Moreover, this conclusion is independent of the value of $\lambda$ since the point $x_{\text {crit }}$ is a stationary point of inflection as one can see from Fig. 2.

\section{Case with fixed scalar charge potential $\Phi$}

Depending on the value of the scalar charge potential, we have two qualitatively different behaviors for $\bar{\beta}$ :

- for $0<\bar{\Phi}<\bar{\Phi}_{c}=1$ : the extremal black hole limit is not allowed inside the cavity and the event horizon radius can therefore take all the possible values inside the cavity, i.e. $0<x<1$. At some temperature, the black hole engulfs the cavity.

- for $\bar{\Phi} \geq \bar{\Phi}_{c}=1$ : the black hole event horizon is bounded from above by the extremal black hole limit that takes place at $x=1 / \bar{\Phi}$. Therefore, at very low temperatures the ensemble is dominated by nearly extremal black holes. In such a case, the black hole can never reach the cavity wall.

In both situations, depending on the value of the event horizon radius, we can have positive or negative scalar charge configurations. The change of sign of $\mathcal{Q}$ takes place at

$$
x_{\mathrm{schw}}=-\frac{1}{2 \bar{\Phi}^{2}}+\frac{1}{2} \sqrt{\frac{1+4 \bar{\Phi}^{2}}{\bar{\Phi}^{4}}},
$$

which is the value where the scalar charge vanishes, turning the hairy black hole into the conventional Schwarzschild black hole. Positive scalar charge configurations are in the domain where $x<x_{\text {schw }}$, implying therefore that black holes with negative scalar charge have $x>x_{\text {schw }}$.

At high temperatures, the ensemble is dominated by black holes with positive scalar charge. As the temperature drops, the scalar charge becomes smaller and the event horizon becomes larger. 
[1] S. Perlmutter et al. [Supernova Cosmology Project Collaboration], Astrophys. J. 517 (1999) 565 astroph/9812133; ; K. Nakamura et al. [Particle Data Group Collaboration], J. Phys. G G 37 (2010) 075021.

[2] C. de Rham, G. Gabadadze and A. J. Tolley, Phys. Rev. Lett. 106 (2011) 231101 arXiv:1011.1232 [hep-th]].

[3] S. F. Hassan and R. A. Rosen, Phys. Rev. Lett. 108 (2012) 041101 arXiv:1106.3344 [hep-th]]; S. F. Hassan, R. A. Rosen and A. Schmidt-May, JHEP 1202 (2012) 026 arXiv:1109.3230 [hep-th]]; S. F. Hassan and R. A. Rosen, JHEP 1204 (2012) 123 [arXiv:1111.2070 [hep-th]].

[4] C. de Rham, G. Gabadadze and A. Tolley, Phys. Lett. B 711 (2012) 190 arXiv:1107.3820 [hep-th]].

[5] N. Arkani-Hamed, H. Georgi and M. D. Schwartz, Annals Phys. 305 (2003) 96 hep-th/0210184.

[6] N. Arkani-Hamed, H.-C. Cheng, M. A. Luty and S. Mukohyama, JHEP 0405 (2004) 074 hepth/0312099.

[7] S. L. Dubovsky, JHEP 0410 (2004) 076 hepth/0409124.

[8] V. A. Rubakov, hep-th/0407104.

[9] D. Blas, D. Comelli, F. Nesti and L. Pilo, Phys. Rev. D 80 (2009) 044025 arXiv:0905.1699 [hep-th]].

[10] S. Dubovsky, P. Tinyakov and M. Zaldarriaga, JHEP 0711 (2007) 083 arXiv:0706.0288 [hep-th]].

[11] D. Comelli, F. Nesti and L. Pilo, Phys. Rev. D 83 (2011) 084042 arXiv:1010.4773 [hep-th]].

[12] Z. Berezhiani, D. Comelli, F. Nesti and L. Pilo, JHEP 0807 (2008) 130 arXiv:0803.1687 [hep-th]]; M. V. Bebronne and P. G. Tinyakov, JHEP 0904 (2009) 100 [Erratum-ibid. 1106 (2011) 018] arXiv:0902.3899 [grqc]]; M. V. Bebronne, arXiv:0910.4066 [gr-qc].

[13] F. Capela and P. G. Tinyakov, JHEP 1104 (2011) 042 arXiv:1102.0479 [gr-qc]].

[14] S. W. Hawking, Phys. Rev. Lett. 26 (1971) 1344; J. D. Bekenstein, Phys. Rev. D 7 (1973) 2333; S. W. Hawking, Commun. Math. Phys. 43 (1975) 199 [Erratum-ibid. 46 (1976) 206].

[15] G. W. Gibbons and S. W. Hawking, Phys. Rev. D 15 (1977) 2752.

[16] S. W. Hawking and D. N. Page, Commun. Math. Phys. 87 (1983) 577.

[17] J. M. Maldacena, Adv. Theor. Math. Phys. 2 (1998) 231 [Int. J. Theor. Phys. 38 (1999) 1113] hep-th/9711200; E. Witten, Adv. Theor. Math. Phys. 2 (1998) 253 hepth/9802150.

[18] E. Witten, Adv. Theor. Math. Phys. 2 (1998) 505 hepth/9803131.

[19] J. W. York, Jr., Phys. Rev. D33 (1986) 2092-2099.

[20] J. W. York, Jr., Phys. Rev. Lett. 28 (1972) 1082.

[21] R. Schon and S. T. Yau, Commun. Math. Phys. 65 (1979) 45; E. Witten, Commun. Math. Phys. 80 (1981) 381;
J. A. Nester, Phys. Lett. A 83 (1981) 241.

[22] N. Arkani-Hamed, H. C. Cheng, M. A. Luty, S. Mukohyama and T. Wiseman, JHEP 0701, (2007) 036 arXiv:hep-ph/0507120.

[23] B. Feldstein, Phys. Rev. D 80 (2009) 044020 arXiv:0904.1212 [hep-th]].

[24] J. D. Brown, J. W. York, Jr., Phys. Rev. D47 (1993) 1407-1419. gr-qc/9209012.

[25] D. Comelli, M. Crisostomi, F. Nesti and L. Pilo, Phys. Rev. D 84 (2011) 104026 [arXiv:1105.3010 [hep-th]].

[26] J. D. Brown and J. W. York, Jr., In *Teitelboim, C. (ed.) et al.: The black hole* 1-24 gr-qc/9405024.

[27] D. J. Gross, M. J. Perry and L. G. Yaffe, Phys. Rev. D 25 (1982) 330.

[28] B. J. Carr, K. Kohri, Y. Sendouda and J. Yokoyama, Phys. Rev. D 81 (2010) 104019 arXiv:0912.5297 [astroph.CO]].

[29] S. L. Dubovsky, P. G. Tinyakov and I. I. Tkachev, Phys. Rev. Lett. 94 (2005) 181102 hep-th/0411158; M. V. Bebronne, arXiv:0905.0819 [gr-qc].

[30] R. M. Wald, Phys. Rev. D 48 (1993) R3427 grqc/9307038.

[31] J. D. Brown, G. L. Comer, E. A. Martinez, J. Melmed, B. F. Whiting and J. W. . York, Class. Quant. Grav. 7 (1990) 1433.

[32] P. Creminelli, A. Nicolis and R. Rattazzi, JHEP 0203 (2002) 051 hep-th/0107141; L. Randall and G. Servant, JHEP 0705 (2007) 054 hep-ph/0607158; G. Nardini, M. Quiros and A. Wulzer, JHEP 0709 (2007) 077 arXiv:0706.3388 [hep-ph]]; B. Hassanain, J. March-Russell and M. Schvellinger, JHEP 0710 (2007) 089 arXiv:0708.2060 [hep-th]]. U. Gursoy, E. Kiritsis, L. Mazzanti and F. Nitti, JHEP 0905 (2009) 033 arXiv:0812.0792 [hep-th]]; T. Konstandin, G. Nardini and M. Quiros, Phys. Rev. D 82 (2010) 083513 arXiv:1007.1468 [hep-ph]];

[33] A. Chamblin, R. Emparan, C. V. Johnson and R. C. Myers, Phys. Rev. D 60 (1999) 064018 hep-th/9902170.

[34] A. P. Lundgren, Phys. Rev. D 77 (2008) 044014 grqc/0612119.

[35] S. Carlip and S. Vaidya, Class. Quant. Grav. 20 (2003) 3827 gr-qc/0306054.

[36] S. L. Dubovsky and S. M. Sibiryakov, Phys. Lett. B 638 (2006) 509 arXiv:hep-th/0603158.

[37] V. Niarchos, Fortsch. Phys. $57 \quad$ (2009) 646 arXiv:0901.2108 [hep-th]].

[38] R. Sundrum, Phys. Rev. D 77 (2008) 086002 arXiv:0708.1871 [hep-th]]. 